\title{
Múltiples formas para segmentar el mercado de compradores online y móvil
}

\author{
Paula RODRÍGUEZ TORRICO \\ Departamento de Economía y \\ Administración de Empresas, Universidad de Burgos. \\ rodrigueztorrico.paula@gmail.com \\ Sonia SAN MARTÍN GUTIÉRREZ \\ Departamento de Economía y \\ Administración de Empresas. \\ Universidad de Burgos. \\ sanmargu@ubu.es \\ Rebeca SAN JOSÉ CABEZUDO \\ Departamento de Organización de Empresas y \\ Comercialización e Investigación de Mercados. \\ Universidad de Valladolid. \\ rebecasc@eco.uva.es
}

Recibido: 02-09-2012

Aceptado: 14-12-2012

\section{RESUMEN}

Dada la situación actual de crecimiento del comercio móvil y online, este proyecto trata de revisar y clasificar las variables que podemos utilizar para caracterizar los perfiles de los compradores en los contextos de compra online y móvil y compararlos. Para ello, recurriremos a características demográficas y socioeconómicas, psicográficas, motivacionales y comportamentales del comprador, supliendo así el gap existente en la literatura sobre el análisis de estas variables.

Palabras clave: Comercio electrónico, Comercio online, Comercio móvil, Segmentación, Perfiles. 


\title{
Multiple ways to segment the market of online and mobile shoppers
}

\begin{abstract}
Given the current growth of mobile and online commerce, this research attempts to review and classify the variables that can be used to characterize the profiles of the buyers and compare them in the context of online and mobile purchasing. To achieve this goal, we will use demographic, socioeconomic, psychographic, behavioral and motivational characteristics of the buyers and thus enhance the literature about the analysis of these variables.
\end{abstract}

Keywords: E-commerce, Online commerce, M-commerce, Segmentation, Profiles.

Sumario: 1. Introducción. 2. La compra online y móvil vs. la compra offline. 3. Variables para caracterizar a los compradores online y móvil: Revisión de literatura. 4. Perfiles de compradores online y móvil. 5. Conclusiones. 6. Implicaciones. Bibliografía

\section{INTRODUCCIÓN}

El entorno online y móvil tiene cada vez más relevancia para el marketing debido a un cambio dado por un nuevo modelo de usuario que consume contenidos y servicios de una manera diferente a como lo venía haciendo hasta ahora (Interactive Advertising Bureau, 2012). Además, el comportamiento de compra online y móvil se transforma en respuesta a la aparición de nuevos canales de compra (p.e. sitios web de bonos y cupones) y debido a las mejoras en el equipamiento de dispositivos tecnológicos con el aumento de la penetración de los smartphones o teléfonos móviles inteligentes, con conexión a Internet y sistema operativo (ONTSI, 2011; Google, 2013).

En el entorno de compra online, la investigación sugiere que los consumidores que compran en este contexto tienen motivaciones diferentes a las de los consumidores que utilizan el canal de compras tradicionales, destacándose en los primeros la búsqueda de comodidad y variedad y en los segundos las compras recreativas (Rohm y Swaminathan, 2004). Además, a partir de la personalidad, confianza y características preferidas de la web también se muestran diferencias entre cada uno de los compradores online (Prodanova y San Martín, 2012). Esto también sucede en el contexto móvil, donde las características únicas de las compras móviles, o m-commerce, (por ejemplo, pantalla pequeña, la capacidad de procesamiento de datos limitados, la ubicuidad, varios tipos de aplicaciones móviles y plataformas diferentes) hacen que los deseos de los consumidores móviles sean diferentes respecto a otros canales de distribución (por ejemplo, de las tiendas físicas, por catálogo y compras online) (Yang et al., 2012). 
A lo anterior se suma el gran crecimiento del comercio a través de estos dos medios digitales (online y móvil) que cada día toman mayor relevancia tanto para las empresas como para los académicos. Según datos publicados por la Comisión del Mercado de las Telecomunicaciones (CMT) este último año (2012) el comercio online ha ingresado 10.455 millones de euros, un 13,6\% más que en el año anterior. Con lo que se constata que el comercio online es una modalidad de comercio joven en plena expansión y crecimiento, incluso en un contexto económico adverso. El número de transacciones llegó a su máximo histórico en el cuarto trimestre del 2012, situándose en los 40 millones de operaciones siendo de 151,6 millones el número de compras en todo el 2012, lo que supone un crecimiento del $15,7 \%$ respecto al año anterior (CMT, 2013). Según el Observatorio Nacional de las Telecomunicaciones y de la Sociedad de la Información (ONTSI), este incremento responde fundamentalmente al incremento del número de internautas compradores (en 2011 se incorporaron 1,8 millones nuevos compradores online). Con relación a su frecuencia de compra existe un 5,6\% de internautas españoles que realiza sus compras online cada semana o quincena. El gasto dedicado a las compras a través de este medio se sitúa en 828 euros de media.

En cuanto al contexto móvil, el último estudio realizado por Google en España (Our Mobile Planet, 2013) destaca la importancia de los smartphones en el cambio de la forma en que los consumidores realizan las compras. Los smartphones se presentan como una herramienta de compra esencial. El 80\% de los consumidores ha buscado un producto o servicio en su dispositivo y esta búsqueda en los teléfonos móviles inteligentes influye en las decisiones del comprador y en las compras en los diferentes medios. Este estudio también informa que el 25\% de los usuarios de smartphones ha comprado a través de su teléfono. Además, según los datos presentados por AIMC en este año, hay un grupo importante de usuarios intensivos de este canal que manifiesta que lo utiliza en más del $60 \%$ de sus compras online, un $19,3 \%$. A esto se unen las compras a través de la tablet, dispositivo electrónico portátil con pantalla táctil y con múltiples prestaciones. Según datos publicados a finales del 2012 por IAB Spain, el 39\% de los usuarios que se conectan mensualmente a Internet a través de la tablet realiza compras al menos una vez al mes a través de este dispositivo. Las previsiones futuras auguran un crecimiento en este sector siendo de 235.400 millones de dólares el valor que se espera de las compras móviles en 2013, un $44 \%$ más que en 2012. Para el periodo 2012-2017 se espera un crecimiento anual del 35\% (Gartner, 2013).

Reflejo de la importancia de estas nuevas formas de distribución, y del incremento del número de compradores online y móvil, existe un interés creciente por identificar a estos compradores, quiénes son y cómo se comportan, tanto en el ámbito académico como en el profesional. Sin embargo la información disponible sobre los perfiles de compradores online y móvil es escasa, siendo segmentado el mercado principalmente a partir de variables demográficas y socioeconómicas, en la mayor parte de los casos. La Tabla 1, elaborada a partir de la información 
secundaria de los estudios consultados muestra esta información para ambos tipos de compradores y en ella se puede observar la similitud entre ellos en cuanto a las características demográficas y socioeconómicas se refiere.

Tabla 1. Perfiles de compradores de acuerdo con información secundaria

\begin{tabular}{l|l|l}
\cline { 2 - 3 } & \multicolumn{1}{c|}{ Comprador online } & \multicolumn{1}{c}{ Comprador móvil } \\
\hline Sexo & Hombre & Hombre \\
\hline Edad & De 25 a 49 años & De 25 a 34 años \\
\hline Área & Localidades con más de 100.000 habitantes o capitales & Ciudad \\
\hline Clase social & Alta/media alta & Ingresos mensuales de 1.801€ a 2.400€ \\
\hline Situación laboral & Trabajadores a tiempo completo & Empleado (jornada completa/parcial) \\
\hline Estado civil & & Soltero/a \\
\hline Fuente: ONTSI (2012), Google (2013) &
\end{tabular}

Ante esta situación de pocas diferencias en perfiles según variables clásicas (ej. como la edad), dada la escasez de literatura académica que analice y compare los distintos tipos de perfiles de compradores en los contextos online y móvil de una forma detallada y con múltiples variables, el propósito principal de este trabajo es realizar una revisión de la literatura existente hasta la fecha sobre las variables caracterizadoras utilizadas en la literatura online y móvil y sobre los perfiles de compradores por ambos medios, para, como fin último, tratar de agrupar las variables a partir de las cuales se puede caracterizar al comprador online y móvil.

\section{LA COMPRA ONLINE Y MÓVIL VS. LA COMPRA OFFLINE}

Durante los últimos 15 años han sido muchos los investigadores que han centrado su atención en el comercio online (Kau et al., 2003; Bhatnagar y Ghose, 2004a, 2004b; Wu y Chou, 2011) por lo que en su definición existe un cierto acuerdo generalizado, pudiendo caracterizarse por el uso de Internet (a partir de un medio online) para vender, comprar e intercambiar bienes y servicios. Se trata de un sistema que facilita y permite la realización de transacciones entre consumidores y empresas (Kotler y Keller, 2006).

Son muchos los autores que han tratado de conocer y observar si existen diferencias entre los compradores que realizan sus compras online y los que no lo hacen, encontrando la mayor parte de ellos en sus resultados diferencias significativas entre los compradores online y los no compradores (Swinyard y Smith, 2003; Rohm y Swaminathan, 2004; Brengman et al., 2005; Allred et al., 2006; Jayawardhena et al., 2007; Brashear et al., 2009). Por ejemplo, Swinyard y Smith (2003) con su análisis comparativo entre compradores y no compradores 
online demuestran que entre ellos existen diferencias significativas en cuanto a su estilo de vida: los compradores son más aventureros en las actividades que realizan en Internet, pasan mayor tiempo tanto en el ordenador como en Internet, son menos temerosos de la posible pérdida financiera en las transacciones online y tienen menor aversión al riesgo. Además, también destacan que ninguno de los dos grupos es homogéneo sino que a su vez se divide en subsegmentos. Brashear et al. (2009) concluyen que los compradores online son más propensos a la búsqueda de comodidad, son innovadores, con menor aversión al riesgo y más impulsivos que los que compran offline.

Son menos los estudios que analizan estas diferencias en el contexto móvil (Bigné et al., 2005; Yang et al., 2012). En este caso, Bigné et al. (2005) encuentran tanto diferencias como similitudes entre los perfiles de compradores y no compradores a través de móvil, no existiendo por ejemplo variaciones en variables como el sexo, el tamaño poblacional o la frecuencia de uso de Internet y situándose la edad, clase social y experiencia de compra online como aquellas que difieren entre los grupos.

En la definición de comercio móvil no existe unanimidad en la literatura al respecto. Algunos autores lo definen en un sentido amplio, otros lo acotan a la mera transacción, algunos lo diferencian del comercio online y para otros es un caso particular de este. A continuación, en la Tabla 2, se observa la variedad de conceptualizaciones que se hacen de la compra por este medio y finalmente se expone la definición propuesta para este trabajo.

Teniendo en cuenta las definiciones anteriores, en este trabajo conceptualizaremos el comercio móvil como la realización de cualquier transacción, que implica la transferencia de la propiedad o derechos de uso de bienes y servicios, que se terminaron mediante el acceso móvil.

Además de lo anterior, el comercio móvil tiene características únicas y diferentes respecto al comercio online. Se da la posibilidad de dirigirse de forma personalizada a los consumidores debido a que el dispositivo pertenece solamente a una persona específica (Choi et al., 2008); permiten la eliminación de barreras espaciales y temporales; gracias a la ubicuidad, las empresas pueden ofrecer servicios de compra a los clientes mientras se desplazan, pudiendo realizar compras en movimiento; y brinda la posibilidad de ofrecer servicios basados en la localización, es decir, ofrecer servicios móviles relevantes en tiempo real al conocer dónde se encuentra el usuario gracias a las tecnologías de GPS para identificar su ubicación física (Shankar y Balasubramanian, 2009; Xu y Yang, 2012; Yang et al., 2012). Una característica que tiene mucha importancia para el marketing es la portabilidad de los smartphones, ya que ofrecen la posibilidad de, por ser inalámbricos y tener un reducido tamaño, llevarlos consigo en todo momento (Choi et al., 2008; Shankar y Balasubramanian, 2009). 
Tabla 2. Definiciones de comercio móvil

\begin{tabular}{|c|c|}
\hline Autores & Definición \\
\hline Durlacher (1999) & $\begin{array}{l}\text { Toda transacción con un valor monetario que se realiza a través de una red de telecomunicaciones } \\
\text { móviles. }\end{array}$ \\
\hline Varshney y Vetter (2002) & $\begin{array}{l}\text { A diferencia del comercio online, el comercio móvil permite que las transacciones se realicen a } \\
\text { través de dispositivos móviles mediante una red de telecomunicaciones inalámbrica. }\end{array}$ \\
\hline $\begin{array}{l}\text { Balasubramanian et al. } \\
(2002)\end{array}$ & $\begin{array}{l}\text { Se define como el uso de la telefonía móvil, los dispositivos inalámbricos (handheld) para } \\
\text { comunicarse y realizar transacciones a través de redes públicas y privadas. }\end{array}$ \\
\hline Lee et al. (2005) & $\begin{array}{l}\text { Comercio móvil en el sentido más amplio puede ser definido como cualquier solicitud de } \\
\text { información o transacción comercial llevada a cabo mediante el uso de dispositivos móviles a través } \\
\text { de una red de comunicación inalámbrica. }\end{array}$ \\
\hline Lin y Wang (2006) & $\begin{array}{l}\text { Los nuevos tipos de transacciones de comercio online, realizadas a través de dispositivos móviles } \\
\text { (por ejemplo, teléfonos móviles, ordenadores portátiles o de tamaño de la palma, y las interfaces, } \\
\text { incluso las instaladas en vehículos), a través de redes de telecomunicaciones inalámbricas y otras } \\
\text { tecnologías de comercio online con cables, se denominan comercio móvil. }\end{array}$ \\
\hline Mahatanankoon (2007) & $\begin{array}{l}\text { El comercio móvil complementa al comercio online permitiendo a los consumidores realizar } \\
\text { transacciones en línea a través de dispositivos móviles (handheld). }\end{array}$ \\
\hline Choi et al. (2008) & $\begin{array}{l}\text { El comercio móvil es igual al comercio online en los tipos de productos y el proceso de transacción. } \\
\text { Los clientes no compran sólo productos/servicios digitales, sino también físicos a través de ambos } \\
\text { canales, los cuales son obtenidos respectivamente mediante descarga online y el proceso de entrega. }\end{array}$ \\
\hline Khalifa et al. (2012) & $\begin{array}{l}\text { Comercio móvil se refiere a la realización de cualquier transacción, que implica la transferencia de la } \\
\text { propiedad o derechos de uso de bienes y servicios, que se iniciaron y/o terminaron mediante el } \\
\text { acceso móvil a redes mediadas por ordenador con la ayuda de un dispositivo electrónico. }\end{array}$ \\
\hline
\end{tabular}

En otro orden, tanto el comercio online como móvil tienen características únicas que los diferencian del canal tradicional de compras (offline), algunas de ellas son comunes a ambos y otras específicas para cada uno. En cuanto aquellas que se dan en los dos medios destacan los ahorros de coste y tiempo; la ausencia de contacto con vendedores, reduciéndose así los posibles enfrentamientos comprador/vendedor; la flexibilidad y la eficiencia, ya que permiten el acceso a una mayor diversidad de productos y tiendas, brindan información en el momento y dan la oportunidad de una comparación de precios de manera instantánea; asimismo, los consumidores tienen un mayor nivel de control en el proceso de compra; aumenta la interactividad entre empresa y consumidor; y, se da la posibilidad para la realización de compras en cualquier lugar del mundo, acabando así con las barreras geográficas. Además, la conveniencia y comodidad toman protagonismo en este tipo de compras, pero también se pueden observar limitaciones como los costes de aprendizaje, la imposibilidad de probar el producto, percepciones de riesgos en cuanto a privacidad e incomodidad con el uso del nuevo medio (Rohm y Swaminathan, 2004; Barnes et al., 2007; Jayawardhena et al., 2007; San Martín Gutiérrez y López Catalán, 2010; Lima-Filho et al., 2012). 


\section{VARIABLES PARA CARACTERIZAR A LOS COMPRADORES ONLINE Y MÓVIL: REVISIÓN DE LITERATURA.}

En la literatura se han utilizado distintos tipos de variables a la hora de caracterizar al comprador móvil y online. Podemos agruparlas a partir de la clasificación de los criterios de segmentación tradicionales e incluir aquellas variables relevantes y diferenciales que puedan servir para caracterizar a los compradores online y móvil (Figura 1). La elección de los criterios tradiciones para segmentar es debido a que dan la posibilidad de agrupar todas las variables oportunas en nuestro caso, y estar extendido su uso.

Figura 1. Variables relevantes de segmentación de mercados de compradores online y móvil

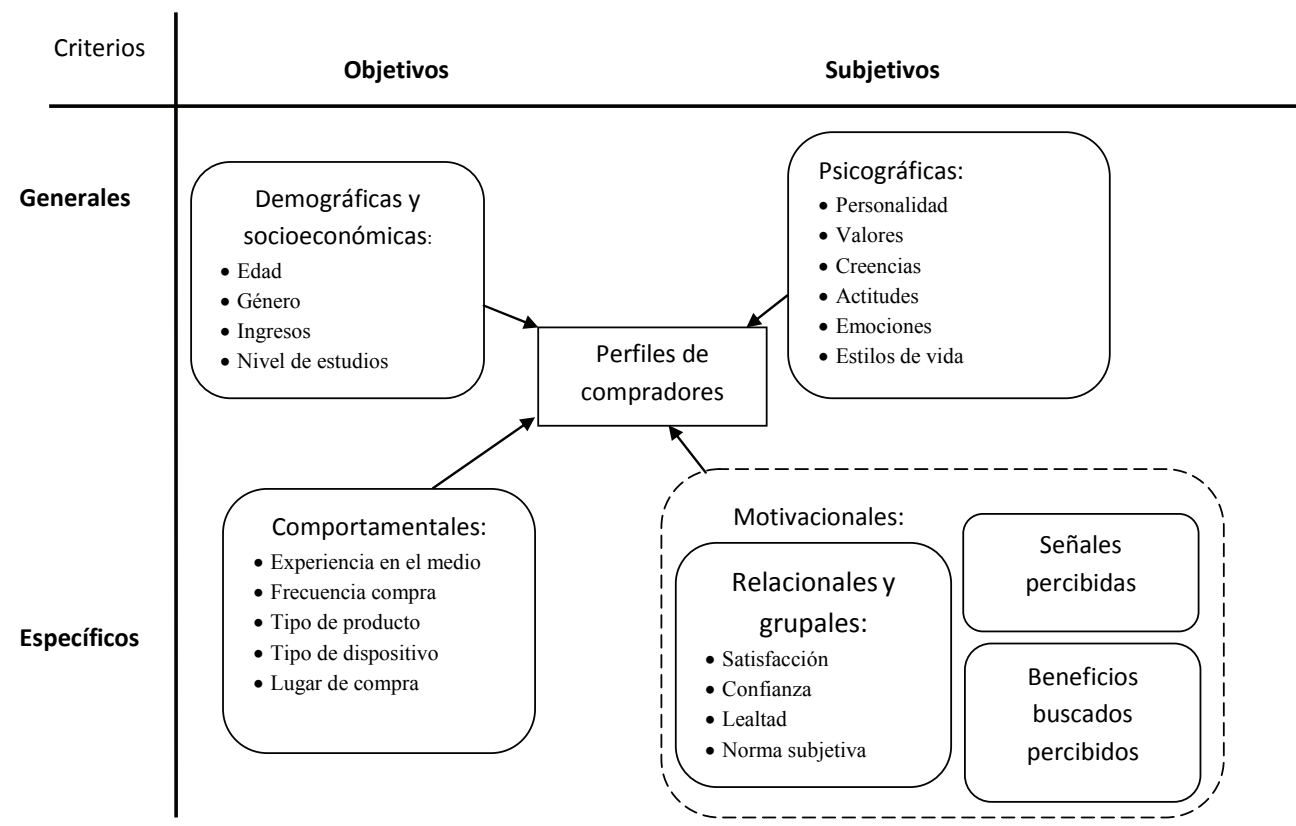

Fuente: Elaboración propia

\section{Variables demográficas y socioeconómicas (Criterios Objetivos/Generales)}

Este grupo de variables hace referencia a características observables del individuo e independientes del tipo de producto o servicio comprado, entre las que se encuentran las características demográficas (edad y género) y las socioeconómicas (ingresos y nivel de estudios). Estas variables han sido las más utilizadas por los autores a la hora de segmentar el mercado, bien sea como variables principales (Bigné et al., 2005; Chong, 2013b) o para conocer más acerca 
del perfil del comprador (Bhatnagar y Ghose, 2004a, 2004b; Yang et al., 2012). No obstante, la investigación acerca del papel que desempeñan en la compra no es concluyente.

- Edad

La edad ha sido una de las variables demográficas más utilizadas a la hora de segmentar el mercado, también en el caso online y móvil. En la literatura se presentan diferencias en cuanto a su importancia. En algunos casos se ha encontrado que la edad afecta de diferente forma en función de la fase del proceso de decisión de compra online (Socer et al., 2005). En el contexto móvil, la edad ha resultado ser una de las variables que mejor predice el comportamiento de compra, siendo los jóvenes los que más uso hacen de él (Ruiz y Sanz, 2007; Bigné et al., 2007, entre otros). Y en otras ocasiones no se han encontrado diferencias significativas respecto a esta variable (Li et al., 2008; Hernández et al., 2011).

- Género

El género ha sido también utilizada para caracterizar al comprador online y móvil. Algunos autores han detectado que los hombres utilizan con mayor frecuencia ambos medios para la compra (Lima-Filho et al., 2012; Yang et al., 2012). En otras ocasiones esta variable no se ha mostrado como un determinante de la adopción en el caso del comercio móvil (Bigné et al., 2005; Li et al., 2008). Li et al. (2008) plantean las dos variables anteriores dentro del Modelo de Adopción Tecnológica (TAM) de Davis (1989) como variables explicativas de la adopción del comercio móvil.

- Ingresos y nivel de estudios

Los ingresos y el nivel de estudios también han mostrado diferencias en cuanto a resultados se refiere. Punj (2011) observó que había variaciones en cuanto a las creencias sobre el comercio online en función del nivel de ingresos y el nivel de estudios del individuo. Pero también existen resultados en los que estas variables no muestran diferencias significativas en los perfiles de compradores online (Moital et al., 2009). En la compra a través del móvil, los ingresos y el nivel de estudios han resultado determinantes en algunas ocasiones (Bigné et al., 2005) y en otras no se ha apreciado su importancia ni en la adopción ni en el comportamiento futuro (Bigné et al., 2007).

Por último, otras variables demográficas como el estado civil (Wu y Chou, 2011) y cultura (Harris et al., 2005) también pueden servir, aunque han sido menos utilizadas dentro de este conjunto. 


\section{Variables psicográficas (Criterios Subjetivos/Generales)}

El estudio de características psicográficas surge ante la necesidad de incluir aspectos psicológicos a la hora de segmentar el mercado, con el fin de conocer cómo se comporta el consumidor (Vyncke, 2002). Dentro de estas características no observables del individuo se encuentran distintos tipos de variables, que son independientes del tipo de producto o servicio que se compre.

\section{- Personalidad}

En primer lugar, las relacionadas con la personalidad del individuo soportadas bajo la Teoría de Difusión de Innovaciones de Rogers (1995). La propensión a la innovación, definida como "un constructo de personalidad que refleja si los individuos están dispuestos a adoptar productos o ideas que son nuevas en el contexto de su experiencia individual" (Aldás Manzano et al., 2008, p.740); la afinidad con el medio entendida como la importancia que tiene un medio en la vida del individuo (Rubin, 1981); la compatibilidad, definida como el grado en que una innovación se percibe como consistente con el valor existente, las experiencias previas y las necesidades de los adoptadores potenciales (Rogers, 1995); la implicación del individuo que hace referencia al estado de interés personal que tiene el individuo en el dispositivo (PC, móvil) como medio de compra-venta (Zaichkowsky, 1985; Moital et al., 2009; San Martín y López Catalán, 2013); la autoeficacia percibida, que aplicado al comercio online, significa que el individuo se siente capaz de buscar información y hacer compras en Internet seguro y cómodo durante la interacción (Hernández et al., 2011); la búsqueda de variedad, que hace referencia a un rasgo del consumidor que hace que alterne entre marcas y productos a pesar de que su selección preferida esté disponible (Ratner y Kahn 2002); la impulsividad es la tendencia de un consumidor a comprar de manera espontánea, no reflexiva, inmediata y cinética (Rook y Fisher, 1995) y la aversión general al riesgo, que es un rasgo personal que difiere de unos individuos a otros, y que se manifiesta como una predisposición a responder de forma negativa ante las situaciones de incertidumbre (Mandrick y Bao, 2005), entre otras.

La literatura ha demostrado que las variables de la personalidad tienen un gran peso en la intención de uso del comercio online (Hernández et al., 2011 y Prodanova y San Martín, 2012) y móvil (Bingé et al., 2007; Aldás-Manzano et al., 2009; Al-Jabri y Sohail, 2012).

- Valores

Los valores y las creencias no han sido variables utilizadas con frecuencia para segmentar a los compradores en estos dos contextos. Los valores se definen como objetivos deseables y circunstanciales que varían en importancia y que sirven como principios rectores en la vida de las personas (Vyncke, 2002). Pascual del 
Riquelme et al. (2011) crearon perfiles de compradores desconfiados y detectaron diferencias en sus valores, siendo más materialistas los desconfiados y presentándose valores de religiosidad en los que menos desconfían. Punj (2011) analizó el efecto moderador de los valores de consumo en la relación de las creencias y el comportamiento de compra online. Para ello se centró en la Teoría de los Valores de Consumo de Sheth, Newman y Gross (1991), la cual afirma que al ir de compras los consumidores tienen en cuenta tanto valores funcionales como hedonistas. Los individuos que se centran en aspectos funcionales suelen considerar más opciones que aquellos que se centran en los hedonistas. La importancia de esta variable recae en que los comportamientos de un individuo pueden estar determinados por su sistema de valores (Chen y Dhillon, 2003).

- Creencias

En cuanto a las creencias, la base teórica sobre la que suele soportarse el estudio de esta variable son la Teoría de Acción Razonada (TRA) de Ajzen y Fishbein (1975, 1980) y la Teoría de Comportamiento Planificado (TPB) de Ajzen (1985). Las creencias se incluyen en estas teorías como principales predictores de las actitudes hacia el uso y la norma subjetiva en el primer caso e incluyendo el control de comportamiento en la segunda teoría. Por lo tanto, podrán determinar la actitud hacia la compra y afectar a variables de influencia social. Punj (2011) desarrolló perfiles de compradores en función de sus creencias hacia las compras online y midió su efecto en el comportamiento de compra moderado por los valores.

- Actitudes

Las actitudes hacia la compra también constituyen un tipo de variable psicográfica (Pedersen, 2005; Brashear et al., 2009; Aldás-Manzano et al., 2009; Hernández et al., 2011) y se definen como la inclinación o la sensación que produce una predisposición a reaccionar favorable o desfavorablemente ante un estímulo (Davis, 1989). Esta variable se incluye en tres teorías de adopción de tecnologías: el Modelo de Adopción Tecnológica (TAM) de Davis (1989), la Teoría de Acción Razonada (TRA) de Fishbein y Ajzen $(1975,1980)$ y la Teoría de Comportamiento Planificado (TPB) de Ajzen (1985). Según estas teorías la intención es el principal predictor de la conducta. En la literatura se ha observado el poder explicativo que tienen las actitudes hacia la intención de comportamiento tanto en el contexto online (Sorce et al., 2005; Hernández et al., 2011) como en el móvil (Pedersen, 2005; Bigné et al., 2007; Aldás-Manzano et al., 2009).

\section{- Emociones}

Las emociones también se han incluido en estos contextos de compra aunque con poca frecuencia. La emoción de consumo hace referencia a "un conjunto de respuestas emocionales provocadas especialmente durante el uso del producto o de la experiencia de consumo" (Gupta y Kim, 2007). Prodanova y San Martín (2012) 
en el contexto online hicieron un análisis de las emociones relacionándolas con los colores, donde encontraron diferencias en la asociación de emociones y colores entre los perfiles creados. En el contexto de compra móvil, Li et al. (2012) analizaron la influencia de las emociones en las experiencias de consumo, dando como resultado una relación positiva entre ambas.

- Estilos de vida

Finalmente, los estilos de vida pueden ser un determinante a la hora de conocer aquellos factores que afectan a la adopción del comercio móvil (López Catalán y San Martín Gutiérrez, 2012). El enfoque AIO (Actividades, Intereses y Opiniones) define los estilos de vida como la manera en la que la gente conduce su vida, incluyendo las actividades, intereses y opiniones (Peter y Olson, 1994). El estilo de vida sirve para caracterizar al comprador online por Swinyard y Smith (2003) y varios autores han replicado su trabajo utilizándola (Brengman et al., 2005; Allred et al., 2006) pero no se ha encontrado en el contexto móvil investigación al respecto. Para ciertos autores, el estilo de vida es el núcleo de la segmentación psicográfica (Vyncke, 2002).

\section{Variables comportamentales (Crietrios Objetivos/Específicos)}

Las variables comportamentales también pueden ser utilizadas para hacer perfiles del comprador online y móvil. En este caso se trata de variables que pueden observarse de forma objetiva y que dependen del proceso de compra o el tipo de producto/servicio comprado. Se pueden distinguir varias en este grupo:

\section{- Experiencia}

La experiencia en el medio es una variable importante que se ha de tener en cuenta, ya que la literatura previa demuestra que en el contexto online predice el aumento de la dependencia hacia el medio (Ruiz y Sanz, 2006). También es una de las que mejor explica el comportamiento positivo hacia las compras móviles (Bigné et al., 2005) e influye de forma significativa en la decisión de compra (Ruiz y Sanz, 2007). Esta variable también ha sido utilizada por autores obtener mayor información sobre el perfil del individuo (Hung et al., 2012).

- Frecuencia de compra

La literatura también ha demostrado el importante papel de la frecuencia de compra en la adopción del comercio móvil aunque no en su intención futura (Bigné et al., 2007; Hernández et al., 2011).

- $\quad$ Tipo de producto

Dependiendo del tipo de producto comprado, tanto en la literatura académica como en estudios desarrollados en el ámbito divulgativo por organismos como ONTSI o $\mathrm{IAB}$, se han encontrado diferencias significativas en las frecuencias de compra 
tanto online como móvil. Rohm y Swaminathan (2004) analizaron la frecuencia de compra entre productos como libros y revistas, hardware, software, servicios de viajes y flores. Bigné et al. (2005) analizaron la frecuencia de los productos comprados a través del móvil y destacaron los tonos de timbre, logos y canciones como los más adquiridos, seguidos de videos, horóscopos y chats. Los autores destacaron la intangibilidad y el bajo coste de estos como justificación de sus resultados. Además, en el último estudio realizado por ONTSI (2012) se muestran tres grupos de productos donde se observan diferencias en cuanto a frecuencia de compra online, existiendo productos/servicios con un alto porcentaje de compras y otros con una frecuencia mínima.

\section{- Tipo de dispositivo y lugar de compra}

Otras variables que se pueden incluir en este grupo, y, que para el contexto de compra móvil pueden tener gran relevancia, son el tipo de dispositivo desde el que se realiza la compra (p.e. smartphone o tablet) y el lugar en el que se realiza. Aunque ambos son móviles, smartphones y tablets tienen características que los hacen muy diferentes desde el punto de vista del marketing. Según datos de Forrester (2013), los usuarios acceden a contenido muy diferente en función de si están utilizando su smartphone o la tablet, haciendo uso del teléfono principalmente para la comunicación y el uso de aplicaciones móvil y la tablet para navegar y consumir medios y contenidos. Los datos de Forrester (2013) también destacan la importancia que tiene el hecho de que los teléfonos móviles son dispositivos personales, a diferencia de las tablets que son compartidas por distintos miembros del hogar, normalmente. Smartphones y tablets también presentan diferencias en cuanto al lugar desde el que se accede. Ambos se utilizan en el hogar pero además los smartphones también se usan en movimiento, es decir, en cualquier lugar en el que se encuentre el individuo pues siempre lo lleva consigo ${ }^{1}$. Este aspecto ha sido poco estudiado en la literatura académica, incluyéndose con el término de ubicuidad cuyo significado indica que los usuarios pueden realizar operaciones de compra móvil en cualquier momento y desde cualquier lugar (Zhou, 2013, p.189). Okazaki y Méndez (2013) crean y validan una escala de ubicuidad formada por cuatro dimensiones -continuidad, inmediatez, portabilidad y capacidad de búsqueda-. En lo que respecta a su contraste, hay escasez de estudios empíricos que lo realicen, Zhou (2013) demostró su influencia en la confianza del consumidor y afirmó que es un aspecto que asegura buenas experiencias de compra móvil en los consumidores. En el campo profesional, los datos de la Mobile Marketing Association (MMA) revelan que un $71 \%$ de los

\footnotetext{
1 A esto se une un nuevo término, "phablet", que hace referencia a aquellos dispositivos que combinan las capacidades del smartphone y la tablet y cuyo tamaño es mayor que el de los primeros pero no llega a tener el tamaño de la tablet.
} 
españoles de entre 25 a 34 años serían más fieles a una determinada marca si recibiesen ofertas exclusivas basadas en su localización.

\section{- Personalización}

Otra variable que puede ser de gran importancia es la personalización, definida como el proceso de generar y presentar el contenido adecuado en el formato correcto para un individuo en el momento adecuado en el lugar correcto (Ho, 2012, p. 803). Los resultados de Shen et al. (2013) destacan que la personalización es una de las variables que afecta en el desarrollo de una imagen positiva de los usuarios hacia la calidad de la información obtenida con los dispositivos móviles.

\section{Variables motivacionales (Criterios Subjetivos/Específicos)}

En este grupo diferenciamos tres subgrupos de variables debido a la dificultad de agruparlas de forma conjunta por las diferencias existentes entre ellas: variables relacionales, beneficios buscados y señales percibidas. Todas ellas no son observables y están relacionadas con el proceso de compra, uso y consumo del producto/servicio comprado. En otras palabras, son las motivaciones que mueven a los individuos a comprar a través de los medios online y móvil.

\section{Variables Relacionales y grupales}

En cuanto a las variables relacionales, se ha detectado el uso en la literatura (online y móvil) de la satisfacción, confianza y lealtad (Lin y Wang, 2006; Prodanova y San Martín, 2012; Hung et al., 2012). Estas tres variables se asientan bajo el enfoque del marketing relacional, definido como "todas las actividades dirigidas a establecer, desarrollar y mantener intercambios relacionales exitosos" (Morgan y Hunt, 1994, p. 22).

\section{- Satisfacción}

Esta variable se refiere al rendimiento que el individuo considera que ha obtenido, refleja el éxito alcanzado durante sus interacciones pasadas e influye en su comportamiento posterior (Raymond, 1990). San Martín y López-Catalán (2013) apuntan que en el caso del comercio móvil, al ser un nuevo medio para muchos consumidores, la satisfacción juega un papel fundamental para el mantenimiento de las relaciones, ya que las primeras experiencias comerciales pueden determinar el futuro de la empresa. Esta variable ha sido utilizada en el contexto de comercio online para medir la satisfacción con distintos servicios (Wu y Chou, 2011) y también Hernández et al. (2011) la utilizan dentro de un modelo ampliado del TAM con consumidores experimentados. 


\section{- Confianza}

La confianza es definida por Morgan y Hunt (1994, p.23) como aquella que existe "cuando una de las partes tiene seguridad en la fiabilidad e integridad de la otra parte del intercambio". Esta variable se ha analizado haciendo referencia a la confianza hacia el proveedor de los servicios de comercio móvil (Chong, 2013a) y hacia la conexión, como una variable dentro del TAM (Li et al., 2008).

- Lealtad

Puede ser definida como un constructo bidimensional que incluye tanto un compromiso actitudinal como una intención de comportamiento de recompra (Lin y Wang, 2006). Esta variable ha sido poco estudiada en el contexto del comercio móvil (Varnali y Toker, 2010), pero tiene una gran importancia, ya que para utilizar de forma eficiente este medio las empresas no sólo deben atraer a los clientes sino que deben retenerlos para garantizar la repetición de la compra, sabiendo que la atracción de clientes en el comercio móvil es más cara que en el comercio tradicional (Lin y Wang, 2006). Algunos autores han tratado la lealtad tanto para el comercio online como móvil (Choi et al., 2008).

- Influencia social

En cuanto al aspecto de la influencia social, fue propuesto en modelos de adopción de tecnologías por Fishbein y Ajzen $(1975,1980)$ en su Teoría de Acción Razonada (TRA) e incluida también en la Teoría del Comportamiento Planeado (TPB), desarrollada por Ajzen (1985) como extensión de la TRA. Según ambas teorías la norma subjetiva es un determinante de la intención de comportamiento y se puede definir como la creencia sobre las consecuencias de la realización de la conducta y de las expectativas normativas de referentes relevantes (Ajzen, 1985, p.14). Refleja la percepción que tiene el individuo acerca de cómo otros piensan que debe comportarse y su motivación para cumplir con las expectativas de los referentes (Fishbein y Ajzen, 1975). La norma subjetiva o influencias sociales han sido aplicadas por los autores a la hora de conocer a los compradores online y móvil (Pedersen, 2005; Khalifa et al., 2012; Li et al., 2012; Chong, 2013a). En comercio móvil, la influencia de terceras personas toma importancia debido a que en algunos casos habrá usuarios que sólo lo utilizarán si hay más usuarios que lo hacen (Chong, 2013a; Khalifa et al., 2012). Pedersen (2005) encontró que la norma subjetiva influía de forma directa en la intención de uso del comercio móvil, así como que las actitudes estaban influidas socialmente.

Beneficios buscados

La segmentación del mercado en función de los beneficios o ventajas buscadas proporciona información muy importante que ofrece facilidades a los vendedores para entender a su audiencia y conocer cómo se comportará (Bhatnagar y Ghose, 2004b). Entre las teorías utilizadas a la hora de analizar las motivaciones o beneficios buscados de los individuos en sus compras están la Teoría de Usos y 
Gratificaciones (Katz et al., 1974), la Teoría de Jerarquía de Necesidades de Maslow (1954) o la Teoría de Motivación (McGuire, 1974). Estas teorías vienen a sugerir que los motivos humanos se orientan principalmente hacia la gratificación y la satisfacción del individuo, proporcionando así la base teórica para estudiar las razones subyacentes por las cuales la gente compra (Rohm y Swaminathan, 2004).

La Teoría de Usos y Gratificaciones fue desarrollada para entender el uso continuado de los medios de comunicación masivos (Katz et al., 1974) pero su empleo se ha extendido para conocer cómo los consumidores toman sus decisiones de compra (Millero, 2003). También se ha utilizado para conocer las motivaciones que llevan a los consumidores a hacer sus compras online (Foucault y Scheufele, 2002; Millero, 2003) y en aspectos relacionados con la adopción del marketing móvil (Nysveen et al, 2005; Sultan et al., 2009).

A continuación, se plantean algunos de los diferentes aspectos buscados en las compras online y móvil que se pueden tener en cuenta a la hora de caracterizar a los compradores:

\section{- Conveniencia}

Hace referencia a la reducción del tiempo dedicado a las compras, flexibilidad en cuanto al momento de hacerlas y menos esfuerzo físico (Rohm y Swaminathan, 2004). Se ha demostrado que es uno de los principales motivos que llevan al consumidor a realizar sus compras online (Rohm y Swaminathan, 2004; Brashear et al., 2009).

\section{- Disfrute percibido}

El disfrute percibido fue planteado por Chong (2013a, 2013b) como un predictor de la adopción y uso de actividades de comercio móvil, debido a que ofrece aplicaciones para juegos, posibilidad de compartir vídeos y utilización de redes sociales.

- Conciencia del precio

La conciencia del precio es otro de los aspectos que pueden incluirse dentro de los beneficios buscados por los compradores a distancia. Puede definirse como la posibilidad de encontrar precios más bajos y además reducir los costes de búsqueda (Brashear et al., 2009).

- Utilidad percibida

Esta variable, definida como el grado en el que una persona cree que usar un determinado sistema podría mejorar su rendimiento (Davis, 1989), puede considerarse un aspecto incluido en los beneficios buscados a la hora de comprar tanto por el medio online como móvil. Es una de las variables principales del TAM (Davis, 1989), en el cual se plantea con una relación directa y positiva con las actitudes e intenciones de comportamiento. El estudio de esta variable ha sido 
objeto de muchas investigaciones en el contexto móvil (Pedersen, 2005; Li et al., 2008; Aldás-Manzano et al., 2009; Hung et al., 2012; Chong, 2013a; Yu y Buahom, 2013) y online (Hernández et al., 2011).

\section{Señales Percibidas}

Por último, hay varias señales que los consumidores utilizan para inferir aspectos del producto o la empresa y les ayudan a decidir en situaciones de información asimétrica (Kirmani y Rao, 2000). En este caso, para agruparlas se utilizará la clasificación realizada por Kirmani y Rao (2000), los cuales distinguen entre dos grupos de señales, aquellas independientes por defecto y las contingentes por defecto; las primeras implican gastos iniciales para la empresa y las segundas posibles gastos futuros. Ambas se subdividen en dos grupos, las primeras en independientes a la venta y contingentes a la venta y las segundas en riesgos en los ingresos y riesgos en los costes.

- Independientes a la venta

En este caso la empresa incurre en un gasto previo y se dan sin necesidad de que se llegue a realizar la transacción (Kirmani y Rao, 2000). En este bloque se encuentra la reputación de la marca, la cual ha sido aplicada tanto en el contexto de compra online como móvil (Brashear et al., 2009; Jiménez Torres y San Martín Gutierrez, 2010). Las relacionadas con la facilidad de uso, utilizadas en modelos de adopción de tecnologías como el TAM tanto en comercio online (Hernández et al., 2011) como en comercio móvil (Aldás-Manzano et al., 2009; Hung et al., 2012). Esta variable es definida como el grado en el que una persona cree que usar un determinado sistema no le supondrá esfuerzo (Davis, 1989). Se ha confirmado su efecto positivo sobre diferentes aspectos como la satisfacción, adopción, actitud e intención de uso (Perdersen, 2005; Aldás-Manzano et al., 2009; Hung et al., 2012; Chong, 2013a). Y, por último, las características extrínsecas del sitio web propuestas por Prodanova y San Martín (2012) que son diseño y reputación del sitio web y que pueden ampliarse con el trabajo de Shen et al. (2013) añadiendo la velocidad y estabilidad de la red inalámbrica y la multifuncionalidad.

\section{- Contingentes a la venta}

Estas señales implican gastos para la empresa pero sólo se dan cuando se realiza la transacción (Kirmani y Rao, 2000). Se incluyen aquí las relacionadas con el servicio al cliente, la interactividad y las promociones, caracterizadas por Prodanova y San Martín (2012) como características relacionales y el acceso a servicios especiales (Jiménez Torres y San Martín Gutierrez, 2010).

- Riesgo en ingresos

Dentro de las señales contingentes por defecto el primer grupo se denomina riesgo en ingresos y hace referencia a la posible pérdida del ingreso potencial si la 
empresa no cumple lo que promete (Kirmani y Rao, 2000). Por lo tanto, aparecen en este bloque señales relacionadas con los contextos de compra online y móvil como son el menor precio (Bhatnagar y Ghose, 2004b; Li et al., 2008), la variedad y mejores opciones (Rohm y Swaminathan, 2004; Moital et al., 2009; Punj, 2011; Chong, 2013a) y el ahorro de tiempo (Punj, 2011).

- Riesgo en costes

Por último, las señales de riesgo en costes, son aquellas señales que en el caso de que la empresa hiciera afirmaciones falsas repercutirían aumentando sus costes (Kirmani y Rao, 2000). En este trabajo se incluyen las características intrínsecas compuestas por la credibilidad de la información, las garantías del sitio desde el que se realiza la compra (Prodanova y San Martín, 2012) y la seguridad percibida (Bhatnagar y Ghose, 2004b; Yu y Buahom, 2013).

A continuación, en la Tabla 3 se resumen todas las variables propuestas para caracterizar a los compradores online y móvil, las fuentes que se han utilizado, el ámbito de estudio (online/móvil) y los marcos teóricos bajo los cuales pueden estudiarse. 
Tabla 3. Resumen de las variables analizadas

\begin{tabular}{|c|c|c|c|}
\hline Variables & Fuentes & $\begin{array}{l}\text { Ámbito de } \\
\text { estudio }\end{array}$ & $\begin{array}{c}\text { Marcos teóricos de referencia o } \\
\text { de utilidad para las variables de } \\
\text { nuestro trabajo }\end{array}$ \\
\hline \multirow[b]{2}{*}{ Edad } & Socer et al. (2005) & Online & \\
\hline & $\begin{array}{l}\text { Ruiz y Sanz (2007), Bigné et al. (2007), Li et al. (2008), } \\
\text { Hernández et al. (2011) }\end{array}$ & Móvil & \\
\hline \multirow[t]{2}{*}{ Género } & Lima-Filho et al. (2012), Yang et al. (2012) & $\begin{array}{l}\text { Online y } \\
\text { móvil }\end{array}$ & \\
\hline & Bigné et al. (2005), Li et al. (2008) & Móvil & \\
\hline \multirow{2}{*}{$\begin{array}{l}\text { Ingresos y nivel de } \\
\text { estudios }\end{array}$} & Moital et al. (2009), Punj (2011) & Online & \\
\hline & Bigné et al. (2005), Bigné et al. (2007) & Móvil & \\
\hline \multirow{2}{*}{ Personalidad } & $\begin{array}{l}\text { Moital et al. (2009), Hernández et al. (2011), Prodanova } \\
\text { y San Martín (2012) }\end{array}$ & Online & \multirow{2}{*}{$\begin{array}{l}\text { Teoría de adopción de } \\
\text { innovaciones de Rogers }\end{array}$} \\
\hline & $\begin{array}{l}\text { Bingé et al. (2007), Aldás-Manzano et al. (2009), Al- } \\
\text { Jabri y Sohail (2012) }\end{array}$ & Móvil & \\
\hline Valores & Pascual del Riquelme et al. (2011), Punj (2011) & Online & $\begin{array}{l}\text { La teoría de los valores de } \\
\text { consumo (Sheth, Newman y } \\
\text { Gross, 1991) }\end{array}$ \\
\hline Creencias & Punj (2011) & Online & $\begin{array}{l}\text { Teoría del comportamiento } \\
\text { planeado (TPB) de Ajzen } \\
(1985) \text {. } \\
\text { Teoría de la acción razonada } \\
\text { (TRA) de Ajzen y Fishbein } \\
(1980)\end{array}$ \\
\hline \multirow[b]{2}{*}{ Actitudes } & Sorce et al. (2005), Hernández et al. (2011) & Online & \multirow{2}{*}{$\begin{array}{l}\text { Teoría del comportamiento } \\
\text { planeado (TPB) de Ajzen } \\
\text { (1985). } \\
\text { Teoría de la acción razonada } \\
\text { (TRA) de Ajzen y Fishbein } \\
\text { (1980) } \\
\text { Modelo de Adopción de } \\
\text { Innovaciones de Davis (1989) }\end{array}$} \\
\hline & $\begin{array}{l}\text { Pedersen (2005), Bigné et al. (2007), Aldás-Manzano et } \\
\text { al. (2009) }\end{array}$ & Móvil & \\
\hline \multirow{2}{*}{ Emociones } & Prodanova y San Martín (2012) & Online & \\
\hline & Li et al. (2012) & Móvil & \\
\hline Estilo de vida & $\begin{array}{l}\text { Swinyard y Smith (2003), Brengman et al. (2005), } \\
\text { Allred et al. (2006) }\end{array}$ & Online & AIO \\
\hline
\end{tabular}


Tabla 4. Resumen de las variables analizadas (continuación)

\begin{tabular}{|c|c|c|c|c|}
\hline \multirow{2}{*}{\multicolumn{2}{|c|}{ Experiencia }} & Ruiz y Sanz (2006) & Online & \\
\hline & & $\begin{array}{l}\text { Bigné et al. (2005), Ruiz y Sanz (2007), Hung et al. } \\
\text { (2012). }\end{array}$ & Móvil & \\
\hline \multicolumn{2}{|c|}{ Frecuencia de compra } & Bigné et al. (2007), Hernández et al. (2011) & Móvil & \\
\hline \multirow{2}{*}{\multicolumn{2}{|c|}{ Tipo de producto }} & Rohm y Swaminathan (2004) & Online & \\
\hline & & Bigné et al. (2005) & Móvil & \\
\hline \multicolumn{2}{|c|}{$\begin{array}{l}\text { Tipo de dispositivo y lugar } \\
\text { de compra }\end{array}$} & Okazaki y Méndez (2013), Zhou (2013) & Móvil & \\
\hline \multicolumn{2}{|c|}{ Personalización } & Ho (2012), Shen et al. (2013) & Móvil & \\
\hline \multirow{2}{*}{\multicolumn{2}{|c|}{ Satisfacción }} & San Martín y López-Catalán (2013) & Online & \multirow{2}{*}{ MK Relacional } \\
\hline & & Wu y Chou (2011), Hernández et al. (2011) & Móvil & \\
\hline \multicolumn{2}{|l|}{ Confianza } & Li et al. (2008), Chong (2013a) & Móvil & MK Relacional \\
\hline \multirow{2}{*}{\multicolumn{2}{|c|}{ Lealtad }} & Choi et al. (2008) & $\begin{array}{l}\text { Online y } \\
\text { móvil }\end{array}$ & \multirow[t]{2}{*}{ MK Relacional } \\
\hline & & Varnali y Toker (2010) & Móvil & \\
\hline \multirow{2}{*}{\multicolumn{2}{|c|}{ Influencia social }} & Pedersen (2005) & Online & \multirow{2}{*}{$\begin{array}{l}\text { Teoría del comportamiento } \\
\text { planeado (TPB) de Ajzen } \\
\text { (1985). } \\
\text { Teoría de la acción razonada } \\
\text { (TRA) de Ajzen y Fishbein } \\
\text { (1980) }\end{array}$} \\
\hline & & Khalifa et al. (2012), Li et al. (2012), Chong (2013a) & Móvil & \\
\hline \multirow{5}{*}{$\begin{array}{l}\text { Beneficios } \\
\text { buscados }\end{array}$} & Conveniencia & Rohm y Swaminathan (2004), Brashear et al. (2009) & Online & \multirow{5}{*}{$\begin{array}{l}\text { Teoría de Usos y } \\
\text { Gratificaciones (Katz et al., } \\
\text { 1974), la Teoría de Jerarquía } \\
\text { de Necesidades de Maslow } \\
\text { (1954) y la Teoría de } \\
\text { Motivación (McGuire, 1974) }\end{array}$} \\
\hline & $\begin{array}{l}\text { Disfrute } \\
\text { percibido }\end{array}$ & Chong (2013a; 2013b) & Móvil & \\
\hline & $\begin{array}{l}\text { Conciencia del } \\
\text { precio }\end{array}$ & Brashear et al. (2009) & Online & \\
\hline & \multirow{2}{*}{$\begin{array}{l}\text { Utilidad } \\
\text { percibida }\end{array}$} & Hernández et al. (2011) & Online & \\
\hline & & $\begin{array}{l}\text { Pedersen (2005), Li et al. (2008), Aldás-Manzano et } \\
\text { al. (2009), Hung et al. 2012), Chong (2013a), Yu y } \\
\text { Buahom (2013) }\end{array}$ & Móvil & \\
\hline \multirow{8}{*}{ Señales } & & $\begin{array}{l}\text { Brashear et al. (2009), Hernández et al. (2011), } \\
\text { Prodanova y San Martín (2012) }\end{array}$ & Online & \multirow{8}{*}{$\begin{array}{l}\text { Teoría de señales Kirmani y } \\
\text { Rao (2000) }\end{array}$} \\
\hline & $\begin{array}{l}\text { Independientes } \\
\text { a la venta }\end{array}$ & $\begin{array}{l}\text { Perdersen (2005), Aldás-Manzano et al. (2009), } \\
\text { Jiménez y San Martín (2010), Hung et al. (2012), } \\
\text { Chong (2013a), Shen et al. (2013) }\end{array}$ & Móvil & \\
\hline & Contingentes & Prodanova y San Martín (2012) & Online & \\
\hline & a la venta & Jiménez y San Martín (2010) & Móvil & \\
\hline & \multirow{2}{*}{$\begin{array}{l}\text { Riesgos en } \\
\text { ingresos }\end{array}$} & $\begin{array}{l}\text { Rohm y Swaminathan (2004), Bhatnagar y Ghose } \\
\text { (2004b), Moital et al. (2009), Punj (2011) }\end{array}$ & Online & \\
\hline & & Li et al. (2008), Chong (2013a) & Móvil & \\
\hline & \multirow{2}{*}{$\begin{array}{l}\text { Riesgos en } \\
\text { costes }\end{array}$} & $\begin{array}{l}\text { Bhatnagar y Ghose (2004b), Prodanova y San Martín } \\
(2012)\end{array}$ & Online & \\
\hline & & Yu y Buahom (2013) & Móvil & \\
\hline
\end{tabular}




\section{PERFILES DE COMPRADORES ONLINE Y MÓVIL}

Algunos autores han encontrado diferencias significativas entre los individuos que realizan sus compras a través de los medios online y móvil y aquellos que las hacen a través de los medios tradicionales u offline, como se expone a continuación.

En cuanto al comercio online, Aljukhadar y Senecal (2011) identifican tres segmentos de compradores online atendiendo a los usos que realizan de Internet: los comunicadores (que utilizan la Red para comunicarse vía online), los compradores al acecho (usan Internet para comprar) y los sociales (los que interactúan en Internet por medio de chats, blogs, etc.). Moital et al. (2009) obtienen cuatro segmentos en función, principalemente, de la implicación que tiene el comprador con el comercio online (entusiastas moderados, compradores reacios, aficionados y no involucrados). Kau et al. (2003) caracterizan a los compradores online utilizando para ello variables comportamentales, actitudinales y de satisfacción y obtienen seis grupos (comprador on-off, comparador, tradicional, dual, rezagado y buscador de información). Cuatro tipologías de compradores online son obtenidas por Rohm y Swaminathan (2004) a partir de sus motivaciones comerciales (comprador de conveniencia, buscador de variedad, comprador equilibrado y comprador orientado a la tienda).

Bhatnagar y Ghose (2004b) segmentan a los compradores de comercio online a partir de los beneficios y riesgos que estos perciben con la compra, obteniendo así tres perfiles (los que observan un alto riesgo de los productos y de seguridad, los que tienen un moderado riesgo del producto y bajo riesgo de seguridad $\mathrm{y}$, por último, aquellos que presentan un bajo riesgo de producto y el riesgo de seguridad es moderado). A partir de las creencias sobre la compra online, Punj (2011) obtuvo tres perfiles (los que creen que ayuda a encontrar la mejor opción y ahorra tiempo, los que creen que ahorra tiempo, y los que creen que ayuda a encontrar la mejor opción). Lima-Filho et al. (2012) realizan una segmentación con base en las características demográficas y socioeconómicas y preferencias, obteniendo cuatro grupos (controlados, compradores jóvenes, compradores básicos y compradores convencionales). Los amantes de las compras, los exploradores aventureros, estudiantes sospechosos y los usuarios empresariales son los perfiles desarrollados por Swinyard y Smith (2003) en función del estilo de vida y su trabajo fue replicado por otros autores (Brengman et al., 2005 y Allred et al., 2006). Jayawardhena et al. (2007) agrupan a los consumidores en función de sus orientaciones hacia la compra (compradores activos, sensibles a los precios, los compradores más exigentes, leales de la marca y orientado hacia la comodidad). Barnes et al. (2007) encuentran tres grupos en función de su personalidad: los escépticos con aversión al riesgo, compradores online de mente abierta y reservados que buscan información. 
En España, Prodanova y San Martín (2012) en función de la personalidad, confianza y las características de la web han desarrollado tres perfiles de compradores online de billetes de transporte: escépticos, reflexivos y prácticos; Pascual del Riquelme et al. (2011) determinaron la existencia de tres grupos de compradores en función de su desconfianza hacia la venta (online y tradicional) y los denominan como consumidores desconfiados en general, consumidores que (específicamente) desconfían de la venta tradicional y consumidores que (específicamente) desconfían de la venta en Internet.

En el contexto móvil, es poca la literatura que hasta la fecha ha tratado de segmentar a los individuos que realizan sus compras a través de su teléfono móvil. La segmentación de los consumidores españoles es realizada por varios autores, Ruiz y Sanz (2007) obtienen nueve segmentos de compradores a través del móvil con las características demográficas y socioeconómicas, las relaciones con el medio y su satisfacción como base de la segmentación; Jiménez y San Martín (2010) destacaron tres grupos de compradores móvil en función de los motivos e inhibidores que llevan a la compra, y los caracterizaron como prácticos, indiferentes y desconfiados; También Bigné et al. (2005) tratan de hacer una aproximación de estos compradores mediante variables demográficas y socioeconómicas y comportamentales sin llegar a desarrollar segmentos.

La información anterior puede verse sintetizada en la Tabla 4, donde se muestran los perfiles de compradores obtenidos en la literatura, las variables principales que han utilizado los autores a la hora de desarrollarlos, así como el ámbito de estudio.

\section{CONCLUSIONES}

En este estudio se realiza una revisión exhaustiva de los trabajos que realizan segmentación del mercado online y móvil así como de las principales variables que pueden utilizarse a la hora de segmentar a los compradores de estos dos medios. Con ello, en primer lugar recomendamos segmentar el mercado a partir de múltiples variables, debido a la inexistencia de investigaciones que hayan realizado de este modo la segmentación del mercado en estos dos contextos.

A partir de esta investigación será posible abordar con el gap existente en la literatura sobre comercio móvil, ya que en la actualidad son pocos los artículos que tratan de caracterizar cómo son los individuos que realizan sus compras a través de este medio, centrándose la mayor parte de la investigación móvil en teorías de adopción de tecnología. 
Tabla 4. Perfiles de compradores detectados

\begin{tabular}{|c|c|c|}
\hline Fuente/Perfiles & Variables & Contexto \\
\hline $\begin{array}{l}\text { Kau et al. }(2003) \\
\text { - Comprador on-off } \\
\text { - Dual } \\
\text { - Comparador } \\
\text { - Rezagado } \\
\text { - Tradicional } \\
\text { - Buscador de información } \\
\end{array}$ & $\begin{array}{c}\text { Género, edad, experiencia, actitudes, } \\
\text { satisfacción }\end{array}$ & Online \\
\hline $\begin{array}{l}\text { Swinyard y Smith (2003) } \\
\text { - Los amantes de las compras } \\
\text { - Los exploradores aventureros } \\
\text { - Estudiantes sospechosos } \\
\text { - Los usuarios empresariales } \\
\end{array}$ & Estilo de vida & Online \\
\hline $\begin{array}{l}\text { Rohm y Swaminathan (2004) } \\
\text { - Comprador de conveniencia } \\
\text { - Buscador de variedad } \\
\text { - Comprador equilibrado } \\
\text { - Comprador orientado a la tienda }\end{array}$ & $\begin{array}{c}\text { Beneficios buscados: } \\
\text { conveniencia } \\
\text { Señales percibidas: riesgo en ingresos }\end{array}$ & Online \\
\hline $\begin{array}{l}\text { Bhatnagar y Ghose (2004b) } \\
\text { - Los que observan un alto riesgo en los } \\
\text { productos y de seguridad } \\
\text { - Los que tienen un moderado riesgo en el } \\
\text { producto y bajo riesgo de seguridad } \\
\text { - Los que presentan un bajo riesgo de } \\
\text { producto y el riesgo de seguridad es moderado }\end{array}$ & $\begin{array}{c}\text { Señales percibidas: riesgo en ingresos } \\
\text { y riesgo en costes }\end{array}$ & Online \\
\hline Bigné et al. (2005) & Edad, género, ingresos, experiencia & Móvil \\
\hline $\begin{array}{l}\text { Jayawardhena et al. (2007) } \\
\text { - Compradores activos } \\
\text { - Sensibles a los precios } \\
\text { - Los compradores más exigentes } \\
\text { - Leales de la marca } \\
\text { - Orientado hacia la comodidad }\end{array}$ & $\begin{array}{l}\text { Orientación de la compra (lealtad, } \\
\text { activo, inactivo, precio y } \\
\text { conveniencia) }\end{array}$ & Online \\
\hline $\begin{array}{l}\text { Barnes et al. (2007) } \\
\text { - Los escépticos con aversión al riesgo } \\
\text { - Compradores online de mente abierta } \\
\text { - Reservados que buscan información }\end{array}$ & Personalidad & Online \\
\hline Ruiz y Sanz (2007) & $\begin{array}{l}\text { Edad, ingresos, nivel de estudios, } \\
\text { género, frecuencia de uso, } \\
\text { experiencia, satisfacción }\end{array}$ & Móvil \\
\hline $\begin{array}{l}\text { Moital } \text { et al. }(2009) \\
\text { - Entusiastas moderados } \\
\text { - Compradores reacios } \\
\text { - Aficionados } \\
\text { - No involucrados } \\
\end{array}$ & $\begin{array}{c}\text { Ingresos y nivel de estudios. } \\
\text { Personalidad (Implicación) } \\
\text { Señales percibidas: riesgos en } \\
\text { ingresos }\end{array}$ & Online \\
\hline $\begin{array}{l}\text { Punj (2009) } \\
\text { - Ayuda a encontrar la mejor opción y ahorra } \\
\text { tiempo } \\
\text { - Ahorra tiempo } \\
\text { - Ayuda a encontrar la mejor opción } \\
\end{array}$ & Creencias & Online \\
\hline $\begin{array}{l}\text { Jiménez y San Martín (2010) } \\
\text { - Prácticos } \\
\text { - Indiferentes } \\
\text { - Desconfiados }\end{array}$ & $\begin{array}{l}\text { Señales percibidas: Independientes y } \\
\text { contingentes a la venta }\end{array}$ & Móvil \\
\hline $\begin{array}{l}\text { Aljukhadar y Senecal (2011) } \\
\text { - Los comunicadores } \\
\text { - Los compradores al acecho } \\
\text { - Los sociales }\end{array}$ & Usos de internet & Online \\
\hline
\end{tabular}


Tabla 4. Perfiles de compradores detectados (continuación)

\begin{tabular}{|l|c|c|}
\hline $\begin{array}{l}\text { Pascual del Riquelme } \text { et al. (2011) } \\
\text { - Desconfiados en general } \\
\text { - Los que (específicamente) desconfian de la } \\
\text { venta tradicional } \\
\text { - Los que (específicamente) desconfian de la } \\
\text { venta en Internet }\end{array}$ & $\begin{array}{c}\text { Valores, personalidad (aversión al } \\
\text { riesgo), desconfianza }\end{array}$ & Online \\
\hline Lima-Filho et al. (2012) & & \\
- Controlados & & \\
- Compradores & Género, edad, ingresos, \\
- Jóvenes & personalidad & Online \\
- Compradores básicos & & \\
- Compradores convencionales & Personalidad, confianza, señales & \\
\hline $\begin{array}{l}\text { Prodanova y San Martín (2012) } \\
\text { - Escépticos }\end{array}$ & percibidas & Online \\
- Reflexivos & & \\
\hline
\end{tabular}

Fuente: Elaboración Propia

Del mismo modo las variables más subjetivas del comprador (p.ej. beneficios buscados) deben tomar un papel protagonista en la caracterización, sin olvidar las relacionales (lealtad, satisfacción y confianza). También, en el contexto móvil las señales percibidas toman gran relevancia dada la novedad y estado inicial de este tipo de compras, las cuales pueden ser una clave para su desarrollo futuro.

Además, sería conveniente ahondar en el estudio de esas variables que hasta la fecha han tenido poco protagonismo en la literatura como son las que hacen referencia a las características propias del dispositivo móvil (ubicuidad, personalización...), analizando su influencia en las actitudes y comportamientos de los compradores, dado que puede ser una línea de investigación rica y novedosa que ofrezca las claves de diferenciación de estos.

Futuros trabajos de investigación podrán comparar las similitudes y diferencias de cada tipo de comprador en función del tipo de compra (online/móvil), así como averiguar la influencia de cada perfil en la intención de compra por uno u otro canal. Otro aspecto interesante sería explorar junto con el medio online y móvil el offline y así realizar un análisis multicanal a partir del cual puede llegarse a conclusiones interesantes sobre todo el proceso de compra.

\section{IMPLICACIONES}

El presente trabajo tiene repercusión tanto para el ámbito académico como profesional, siendo su contribución académica triple. En primer lugar, se propone una clasificación detallada de variables que permitirá hacer una segmentación del mercado online y móvil a partir de todas las variables relevantes en ambos contextos, algo que no se ha detectado en la literatura. En segundo lugar, se cubre 
el gap existente hasta la fecha en la segmentación de compradores a través del móvil, dando una visión completa de cómo segmentar a los individuos que realizan sus compras a través de este dispositivo. Por último, no sólo se contribuye con información sobre un medio sino que se dan las bases necesarias para hacer perfiles de compradores online y móvil, por lo que puede ayudar a crear sinergias entre ambos medios y averiguar si el target puede ser el mismo en los dos casos o es muy distinto.

Normalmente las empresas tienden a utilizar criterios objetivos generales para segmentar su mercado por su facilidad y accesibilidad a los datos, pero estos criterios no se adaptan hoy día a la personalización que demanda el mercado y a un marketing de nichos más que de grandes segmentos. No obstante, hay que reconocer la dificultad para las empresas de obtener información primaria en muchas ocasiones sobre algunos de los criterios de segmentación que proponemos y que son subjetivos o específicos, pero que probablemente sea más rentable en el largo plazo hacerlo así para diferenciarse a pesar de los avances en este sentido gracias a la relación con los clientes a través de las redes sociales como Facebook, Twitter, etc.

Por ello, en el campo profesional el trabajo proporciona información acerca de cómo segmentar a los consumidores a partir no sólo de criterios objetivos generales, lo que contribuirá prestando ayuda a los gerentes de marketing a la hora de dirigir sus productos, campañas y acciones al mercado de forma más segmentada y con un mayor conocimiento de sus clientes. Tras la segmentación, la empresa debe tener los recursos y debe diseñar estrategias adaptadas a esos grupos de consumidores objetivos y ser las pioneras o líderes en esos segmentos, máxime en un mercado como el del m-commerce que está comenzando. Del mismo modo, la comparación de los perfiles de compradores online y móvil puede prestar información a las empresas para dirigirse de una u otra forma en cada uno de los medios, pudiendo obtener resultados interesantes en este aspecto en el que se observen sinergias entre los dos contextos de compras y proporcionen claves para el desarrollo de estrategias multicanal (o cross-media) más eficaces.

Queremos finalizar señalando la dificultad de clasificar múltiples variables que pueden servir para caracterizar perfiles en un contexto de compra como el móvil, sobre el que la investigación es aún escasa, máxime en España, donde este tipo de compra está aún en fase de lanzamiento. Es posible que hayamos omitido alguna variable que también pueda servir para caracterizar perfiles, lo que puede ser una posible limitación del trabajo. Este artículo supone un reto que contribuirá a ayudar a las empresas que estén reflexionando sobre la introducción de su negocio en la venta por móvil, de forma exclusiva o complementaria a la compra online y a las que ya se han lanzado en este campo y únicamente han realizado una segmentación clásica de sus compradores potenciales. 


\section{BIBLIOGRAFÍA}

AJZEN, I. y FISHBEIN, M., Understanding Attitudes and Predicting Social Behavior, Englewood Cliffs, NJ: Prentice-Hall, 1980, ISBN: 0139364358

ALDÁS-MANZANO, J., RUIZ-MAFÉ, C. y SANZ-BLAS, S., Exploring individual personality factors as drivers of $\mathrm{M}$-shopping acceptance, Industrial Management \& Data Systems, Vol. 109, no 6, 2009, p. 739-757.

AL-JABRI, I.M Y SOHAIL, M.S., Mobile Banking Adoption: Application Of Diffusion Of Innovation Theory, Journal of Electronic Commerce Research, Vol. 13, no 4, 2012, p. 379-391.

ALJUKHADAR, M., y SENECAL, S., Segmenting the online consumer market, Marketing Intelligence \& Planning, Vol. 29, no 4, 2011, p. 421-435.

ALLRED, C., SMITH, S.M. y SWINYARD, W.R., Shopping lovers and fearful conservatives: a market segmentation analysis, International Journal of Retail \& Distribution Management, Vol. 34, no 4/5, 2006, p. 308-34.

Asociación para la Investigación de Medios de Comunicación 2013. Encuesta AIMC a usuarios de internet, http://www.aimc.es/

BALASUBRAMANIAN, S., PETERSON, R.A. y JARVENPAA, S.L., Exploring the Implications of M-commerce for Markets and Marketing, Journal of the Academy of Marketing Science, Vol. 30, no 4, 2002, p. 348361.

BARNES, S.J., BAUER, H.H., NEUMANN, M.M. y HUBER, F., Segmenting cyberspace: a customer typology for the internet, European Journal of Marketing, Vol. 41, no 1/2, 2007, p. 71-93.

BHATNAGAR, A., y GHOSE, S., A latent class segmentation analysis of eshoppers, Journal of Business Research, Vol. 57, no 7, 2004a, p. 758-767.

BHATNAGAR, A., y GHOSE, S., Segmenting consumers based on the benefits and risks of internet shopping, Journal of Business Research, Vol. 57, no 12, 2004b, p. 1352-1360.

BIGNÉ, E., RUIZ, C., y SANZ, S., The impact of internet user shopping patterns and demographics on consumer mobile buying behavior, Journal of Electronic Commerce Research, Vol. 6, no 3, 2005, p. 193-209.

BIGNÉ, E., RUIZ, C., y SANZ, S., Key drivers of mobile commerce adoption. an exploratory study of spanish mobile users, Journal of Theoretical and Applied Electronic Commerce Research, Vol. 2, no 2, 2007, p. 48-60.

BRASHEAR, T. G., KASHYAP, V., MUSANTE, M. D., y DONTHU, N., A profile of the internet shopper: Evidence from six countries, Journal of Marketing Theory and Practice, Vol. 17, no 3, 2009, p. 267-281.

BRENGMAN, M., GEUENS, M., WEIJTERS, B., SMITH, S.M. y SWINYARD, W.R., Segmenting internet shoppers based on their web-usage-related lifestyle: a cross-cultural validation, Journal of Business Research, Vol. 58, no 1, 2005, p. 79-88. 
CHEN, S.C. y DHILLON, G.S., Interpreting Dimensions of Consumer Trust in E-Commerce, Information Technology and Management, Vol. 4, no 2/3, 2003, p. 303-318.

CHOI, J., SEOL, H., LEE, S., CHO, H., y PARK, Y., Customer satisfaction factors of mobile commerce in Korea, Internet Research, Vol. 18, no 3, 2008, p. 313-335.

CHONG, A. Y. A two-staged SEM-neural network approach for understanding and predicting the determinants of m-commerce adoption, Expert Systems with Applications, Vol. 40, no 4, 2013a, p. 1240-1247.

CHONG, A. Y., Mobile commerce usage activities: The roles of demographic and motivation variables, Technological Forecasting and Social Change, disponible online 15 enero 2013, 2013b.

Comisión del Mercado de las Telecomunicaciones 2013, Informe sobre el comercio electrónico en España a través de entidades de medios de pago. Cuarto trimestre 2012, http://www.cmt.es/

DAVIS, F.D., Perceived usefulness, perceived ease of use and user acceptance of information technology, MIS Quaterly, Vol. 13, no 3, 1989, p. 319-40.

Durlacher Research. 1999, Mobile commerce report, London: Durlacher Research.

FISHBEIN, M. y AJZEN, I., Belief, Attitude, Intention and Behavior: An Introduction to Theory and Research, Reading, MA: Addison-Wesley, 1975, ISBN: 9780201020892

FOUCAULT, B.E. y SCHEUFELE, D.A., Web vs campus store? Why students buy textbooks online, The Journal of Consumer Marketing, Vol. 19, no 4/5, 2002, p. 409-423.

Gartner 2013. Forecast: Mobile Payment, Worldwide, 2013 Update, http://www.gartner.com/technology/home.jsp

Google 2012. Our Mobile Planet: España. Conoce mejor al consumidor móvil, http://services.google.com/fh/files/blogs/our_mobile_planet_spain_es.pdf

GUPTA, S. y KIM, H.W., The moderating effect of transaction experience on the decision calculus in on-line repurchase, International Journal of Electronic Commerce, Vol. 12, no 1, 2007, p. 127-58.

HARRIS, P., RETTIE, R., y CHEUNG, C. K., Adoption and usage of mcommerce: A cross-cultural comparison of Hong Kong and the United Kingdom, Journal of Electronic Commerce Research, Vol. 6, no 3, 2005, p. 210-224.

HERNÁNDEZ, B., JIMÉNEZ, J. y MARTÍN, M. J., Age, gender and income: do they really moderate online shopping behaviour?, Online Information Review, Vol. 35, no 1, 2011, p. 113-133.

$\mathrm{HO}, \mathrm{S}$. Y. The effects of location personalization on individuals' intention to use mobile services, Decision Support Systems, Vol. 53, no 4, 2012, p. 802. 
HUNG, M., YANG, S., y HSIEH, T., An examination of the determinants of mobile shopping continuance, International Journal of Electronic Business Management, Vol. 10, no 1, 2012, p. 29-37.

Informe Forrester 2013, The Smartphone \& Tablet Experience, http://www.forrester.com/home.

Interactive Advertising Bureau 2011, III Estudio IAB Spain sobre Mobile Marketing. Noviembre de 2011, http://www.iabspain.net/

Interactive Advertising Bureau 2012, IV Estudio IAB Spain sobre Mobile Marketing: Informe de Resultados Septiembre de 2012, http://www.iabspain.net/

JAYAWARDHENA, C., WRIGHT, L.T. y DENNIS, C., Consumers online: intentions, orientations and segmentation, International Journal of Retail \& Distribution Management, Vol. 35, no 6, 2007, p. 515-26.

JIMÉNEZ TORRES, N.H. y SAN MARTÍN GUTIÉRREZ, S., ¿Por qué compra por teléfono móvil el consumidor español?, Boletín económico del ICE $\mathrm{n}^{\circ}$ 2987, $2010 \quad \mathrm{http} / / / w w w . r e v i s t a s i c e . c o m / C a c h e P D F / B I C E \_2987 \_11-$ 22 B5535C7D9EEECC755636F0783B368D97.pdf

KATZ, E., BLUMLER, J. y GUREVITCH, M., Utilization of mass communication by the individual, En: BLUMLER, J. y KATZ. E. The uses of mass communications: Current perspectives on gratifications research. Beverly Hills: Sage, 1974, p. 19-32. ISBN: 9780803903401

KAU, A. K., TANG, Y. E. y GHOSE, S., Typology of online shoppers, The Journal of Consumer Marketing, Vol. 20, no 2/3, 2003, p. 139-156.

KHALIFA, M., CHENG, S. K. N., y SHEN, K. N., Adoption of mobile commerce: A confidence model, The Journal of Computer Information Systems, Vol. 53, no 1, 2012, p. 14-22.

KIRMANI, A. y RAO, A.R., No Pain, No Gain: A Critical Review of the Literature on Signaling Unobservable Product Quality, Journal of Marketing, Vol. 64, 2000, p. 66-79.

KOTLER, P. y KELLER, K.L., Marketing Management, 12e Prentice-Hall, 2006, ISBN: 9780131457577

LEE I., KIM J. y KIM J., Use contexts for the mobile internet: A longitudinal study monitoring actual use of mobile internet services, International Journal of Human-Computer Interaction, Vol. 18, no 3, 2005, p. 269-292.

LI, M., DONG, Z. Y. y CHEN, X., Factors influencing consumption experience of mobile commerce, Internet Research, Vol. 22, no 2, 2012, p. 120-141.

LI, S., GLASS, R., y RECORDS, H., The influence of gender on new technology adoption and use-mobile commerce, Journal of Internet Commerce, Vol. 7, no 2, 2008, p. 270-289.

LIMA-FILHO, D. D. O., ALVES, C. D. S., QUEVEDO-SILVA, F., MOREIRA, L. B., GARCEZ, V. R., y ARATANI, W. F., Profile of the electronic 
commerce consumer: A study with brazilian university students, Journal of Internet Banking and Commerce, Vol. 17, no 1, 2012, p. 1-16.

LIN, H.H. y WANG, Y.S., An examination of the determinants of customer loyalty in mobile commerce contexts, Information \& Management, Vol. 43, no 3, 2006, p. 271-82.

LÓPEZ CATALÁN, B. y SAN MARTÍN GUTIÉRREZ, S., Enfoques en el estudio de la adopción de las compras por teléfono móvil en los particulares, Cuadernos Aragoneses de Economía. $2^{\text {a }}$ Época, Vol. 22, no 1-2, 2012, p. 151173.

MAHATANANKOON, P., The effects of personality traits and optimum stimulation level on text-messaging activities and m-commerce intention, International Journal of Electronic Commerce, Vol. 12, no 1, 2007, p. 7-30.

MANDRICK, C.A. y BAO, Y., Exploring the Concept and Measurement of General Risk Aversion, Advances in Consumer Research, Vol. 32, no 1, 2005, p. 531-539.

MCGUIRE W. Psychological motives and communication gratification, En: BLUMLER JF, KATZ J. The uses of mass communications: current perspectives on gratification research. Beverly Hills CA: Sage. 1974, p. 10667. ISBN: 9780803903401

MILLERO, M.R. 2003, E-commerce uses and gratifications and framing study on media portrayal and perspectives of the retail e-commerce Web site industry, West Virginia University, ProQuest, UMI Dissertations Publishing. 1422079.

Mobile Marketing Association, III Estudio de Comercio Móvil, 2013, http://mmaspain.com/

MOITAL, M., VAUGHAN, R., y EDWARDS, J., Using involvement for segmenting the adoption of e-commerce in travel, Service Industries Journal, Vol. 29, no 5, 2009, p. 723-739.

MORGAN, R.M. y HUNT, S.D., The commitment-trust theory of relationship marketing, Journal of Marketing, Vol. 58, no 7, 1994, p. 20-38.

NYSVEEN, H., PEDERSEN, P.E. y THORBJORNSEN, H., Intentions to Use Mobile Services: Antecedents and Cross-Service Comparisons, Academy of Marketing Science Journal Vol. 33, no 3, 2005, p. 330-346.

Observatorio de las Telecomunicaciones y la Sociedad de la Información Ministerio de Industria, Comercio y Turismo 2012. Comercio electrónico B2C 2011, http://observatorio.red.es/

OKAZAKI, S. Y MÉNDEZ, F., Perceived Ubiquity in Mobile Services, Journal of Interactive Marketing Vol. 27, no 2, 2013, p. 98.

PASCUAL DEL RIQUELME, M.I., ROMÁN-NICOLAS, S. y RODRÍGUEZHERRERA, R., Desconfianza hacia la venta tradicional y electrónica: Un estudio sobre el perfil del consumidor desconfiado, Universia Business Review. Tercer trimestre 2011. 
PEDERSEN, P. B., Adoption of mobile intemet services: An exploratory study of mobile commerce early adopters, Journal of Organizational Computing and Electronic Commerce, Vol. 15, no 2, 2005, p. 203-222.

PETER, J.P. y OLSON J.C., Understanding Consumer Behavior. Burr Ridge, IL: Irwin, 1994. ISBN: 0256122784

PRODANOVA, J. y SAN MARTÍN, S. Todos los compradores online de billetes de transporte no son iguales, Boletín económico del ICE no 3029, 2012, http://www.revistasice.com/CachePDF/BICE_3029_53_3D016EF0B59B4F DFEA182F03DDE69E86.pdf

PUNJ, G., Effect of Consumer Beliefs on Online Purchase Behavior: The Influence of Demographic Characteristics and Consumption Values, Journal of Interactive Marketing. Vol. 25, no 3, 2011, p. 134-144.

RATNER, R. K. y KAHN, B. E., The Impact of Private versus Public Consumption on Variety-Seeking Behavior, Journal of Consumer Research, Vol. 29, no 2, 2002, p. 246-257.

RAYMOND, G.A., Problems and prospects in the study of international norms, The International Studies Review, Vol. 41, no 2, 1990, p. 205-45.

ROGERS, E., Diffusion of Innovations, 4th ed., The Free Press, New York, NY, 1995.

ROHM, A. J., y SWAMINATHAN, V., A typology of online shoppers based on shopping motivations, Journal of Business Research, Vol. 57, no 7, 2004, p. 748-757.

ROOK, D.W. y FISHER, R.J., Trait and normative aspects of impulsive buying behavior, Journal of Consumer Research, Vol. 22, no 3, 1995, p. 305-13.

RUBIN, A.M., An examination of television viewing motivations, Communications Research, Vol. 8, 1981, p. 141-65.

RUIZ, C. y SANZ, S., Explaining Internet dependency. An exploratory study of future purchase intention of Spanish Internet users, Internet Research, Vol. 16, no 4, 2006, p. 380-397.

RUIZ, C. y SANZ, S., Tipología de compradores a través del teléfono móvil un estudio de segmentación de los consumidores españoles, Dirección y Organización: Revista de Dirección, Organización y Administración de Empresas, Vol. 34, 2007, p. 44-52.

SAN MARTÍN GUTIÉRREZ, S y LÓPEZ CATALÁN, B., Posibilidades de la compraventa B2C por teléfono móvil en comparación con internet, Cuadernos de gestión, Vol. 10, no 1, 2010, p. 17-34.

SAN MARTÍN, S y CAMARERO, C., Consumer trust to a web site. Moderating efect of attitudes towards online shopping, Cyberpsychology \& Behavior. Vol. 11 , no 5, 2008, p. 549-554.

SAN MARTÍN, S. y LÓPEZ CATALÁN, B., How can a mobile vendor get satisfied customers? Industrial Management \& Data Systems. Vol. 113, no 2, 2013, p. 156-170. 
SHANKAR, V. y BALASUBRAMANIAN, S., Mobile Marketing: A Synthesis and Prognosis, Journal of Interactive Marketing, Vol. 23, no 2, 2009, p. 118129.

SHEN X.L., WANG, N., SUN, Y. y XIANG, L., Unleash the power of mobile word-of-mouth: An empirical study of system and information characteristics in ubiquitous decision making, Online Information Review, Vol. 37, no 1, 2013, p. 42-60.

SHETH, J., NEWMAN, B.I., y GROSS, B.L., Consumption Values and Market Choices: Theory and Applications, Cincinnati: South-Western Publishing, 1991. ISBN: 0538805633

SORCE, P., PEROTTI, V., y WIDRICK, S., Attitude and age differences in online buying, International Journal of Retail \& Distribution Management, Vol. 33, no 2/3, 2005, p. 122-132.

SUlTAN, F., ROHM, A. J. y GAO, T., Factors Influencing Consumer Acceptance of Mobile Marketing: A Two-Country Study of Youth Markets, Journal of Interactive Marketing Vol. 23, no 4, 2009, p. 308-320.

SWINYARD, W.R. y SMITH, S.M., Why people don't shop online: a lifestyle study of the internet consumer, Psychology \& Marketing, Vol. 20, no 7, 2003, p. 567-97.

VARNALI, K. y TOKER, A., Mobile marketing research: The-state-of-the-art, International Journal of Information Management, Vol. 30, 2010, p. 144-151

VARSHNEY, U., y VETTER, R., Mobile commerce: Framework, applications and networking support, Mobile Networks and Applications, Vol. 7, no 3, 2002, p. 185-198.

VYNCKE, P., Lifestyle Segmentation: From Attitudes, Interests and Opinions, to Values, Aesthetic Styles, Life Visions and Media Preferences, European Journal of Communication, Vol. 17, no 4, 2002, p. 445-463.

WU, R-S. y CHOU, P.C., Customer segmentation of multiple category data in ecommerce using a soft-clustering approach, Electronic Commerce Research and Applications, Vol. 10, no 3, 2011, p. 331-341.

YANG, K., y HYE-YOUNG, K., Mobile shopping motivation: An application of multiple discriminant analysis, International Journal of Retail \& Distribution Management, Vol. 40, no 10, 2012, p. 778-789.

YU, Y-W., Y BUAHOM, K., Exploring Factors Influencing Consumer Adoption on Mobile Commerce Services, The Business Review, Cambridge, Vol. 21, no 1, 2013, p. 258-265.

ZAICHKOWSKY, J.L., Measuring the involvement construct, Journal of Consumer Research, Vol. 12, 1985, p. 341-51

ZHOU, T., An empirical examination of the determinants of mobile purchase, Personal Ubiquity Computer, Vol. 17, 2013, p. 187-195 